\title{
Antipsychotic Use in Seniors
}

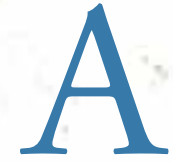

ntipsychotics were primarily developed for the treatment of schizophrenia and bipolar disorder, but they are increasingly being used to treat the behavioural and psychological symptoms associated with dementia in seniors, such as delusion, aggression and agitation.

- The prevalence of dementia in Canada has been estimated at $2.5 \%$ among seniors between the ages of 65 and 74 , compared to $34.5 \%$ among those 85 and older.

- Total drug program expenditures on antipsychotics based on paid claims in five provinces (Alberta, Saskatchewan, Manitoba, New Brunswick and Nova Scotia) increased at an average annual rate of $13.1 \%$ over the study period, accounting for $2.0 \%$ of total drug program expenditures on seniors in 2006-2007.

- Atypical antipsychotics accounted for the highest proportion of drug program antipsychotic expenditure in 2006-2007 (94.5\%), with typical antipsychotics making up the remainder (5.5\%).

- The age-sex standardized rate of antipsychotic use among seniors on public drug programs increased in each of the five provinces during the study period, with the overall rate of use increasing from $4.3 \%$ in 2001-2002 to 5.0\% in 2006-2007.

- The rate of growth of antipsychotic use slowed during the study period, from an average of $5.2 \%$ per year between 2001-2002 and 2003-2004 to 1.0\% between 2003-2004 and 2006-2007. The decline in growth followed the release of three regulatory warnings related to the use of antipsychotics in seniors with dementia.

- The lowest rate of antipsychotic use was among seniors between the ages of 65 and 74 (3.0\% in males and $3.6 \%$ in females), and the highest rate was found in those 85 and older (9.1\% in males and $11.4 \%$ in females). The higher use in older seniors is likely to due, in part, to the fact that the prevalence of dementia increases with age.
- The average daily dose of atypical antipsychotics used by seniors on public drug programs was highest among those age 65 to 74 , and lowest among those age 85 and older.

- Risperidone had the highest rate of use among the atypical antipsychotics in every year between 2001-2002 and 2006-2007. Olanzapine had the second highest rate of use among seniors in the first four years of the study period, but was surpassed by quetiapine in both 2005-2006 and 2006-2007.

- In 2006-2007, 37.7\% of senior nursing home residents on public drug programs in Manitoba, New Brunswick and P.E.I. had claims for antipsychotics, compared to only $2.6 \%$ of senior claimants living in the community. The higher rate of antipsychotic use in nursing homes is likely due, in part, to a higher prevalence of dementia among nursing home residents. HQ

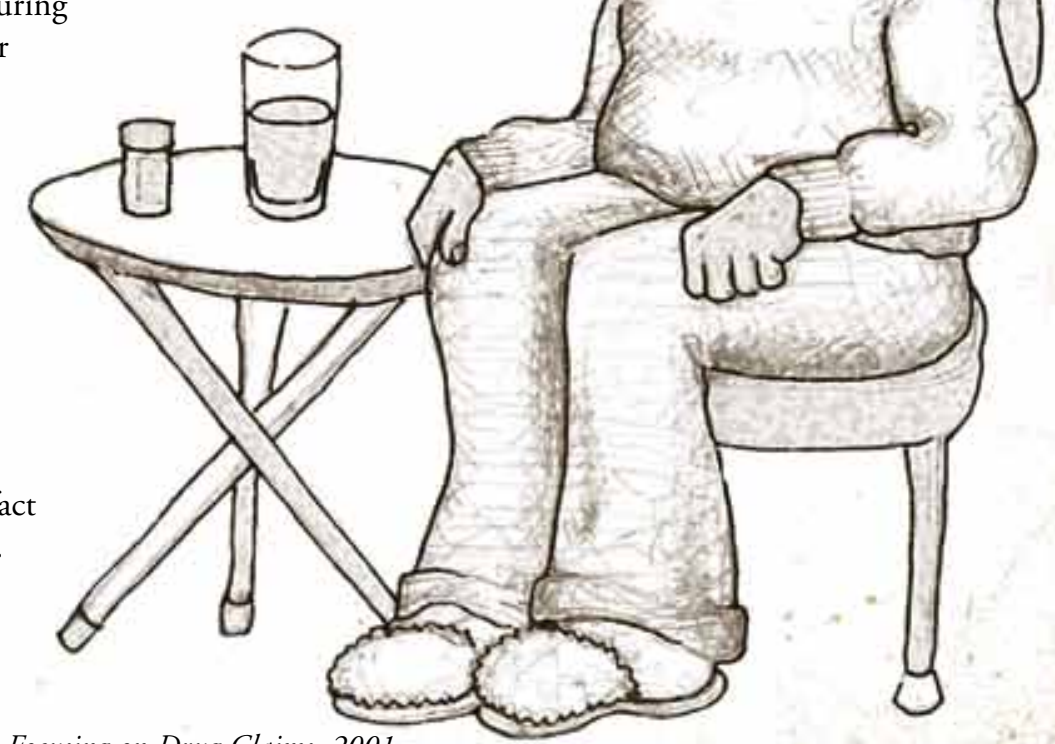

Facts taken from Antipsychotic Use in Seniors: An Analysis Focusing on Drug Claims, 2001 to $2007 \mathrm{http}: / /$ secure.cihi.ca/cihiweb/dispPage.jsp?cw_page=AR_2970_E\&cw_topic=2970 